\title{
ON UNIT GROUPS AND CLASS GROUPS OF QUARTIC FIELDS OF SIGNATURE $(2,1)$
}

\author{
J. BUCHMANN, M. POHST, AND J. GRAF V. SCHMETTOW
}

\begin{abstract}
This is the third and last paper of a series, now completing the description of the unit group and class group of all quartic number fields $F$ of discriminant $d_{F}$ with $\left|d_{F}\right|<10^{6}$.
\end{abstract}

\section{INTRODUCTION}

In this paper we consider quartic fields $F$ with two real conjugates. Using the tables of David Ford and the first two authors [2], we computed unit groups $U_{F}=\langle-1\rangle \times\left\langle\varepsilon_{1}\right\rangle \times\left\langle\varepsilon_{2}\right\rangle$ and class groups $C l_{F}$ of all 90671 number fields $F$ whose discriminant $d_{F}$ is bounded in absolute value by one million. A comparison shows that our results are not in precise agreement with the predictions of Cohen and Martinet [4]. However, this was not to be expected because of the relatively small range of discriminants under consideration.

After [3] and [5], this paper completes the description of the most important invariants of all quartic number fields $F$ with $\left|d_{F}\right|<10^{6}$.

\section{UNIT GROUPS}

The fundamental units were computed by using the generalized Voronoi algorithm [1]. The algorithm operates as follows: For $\alpha \in F$, let $\alpha^{(1)}, \alpha^{(2)}$ be the real conjugates of $\alpha$, and $\alpha^{(3)}, \alpha^{(4)}$ the nonreal, complex conjugates; i.e., $\alpha^{(4)}$ is complex conjugate to $\alpha^{(3)}$. We set

$$
\begin{aligned}
& |\alpha|_{1}:=\left|\alpha^{(1)}\right|, \\
& |\alpha|_{2}:=\left|\alpha^{(2)}\right|, \\
& |\alpha|_{3}:=\left|\alpha^{(3)}\right|^{2} .
\end{aligned}
$$

A fractional ideal a in $F$ is called reduced if

$$
1 \in \mathbf{a} \text { and }\left\{\alpha \in \mathbf{a}:|\alpha|_{i}<1,1 \leq i \leq 3\right\}=\{0\} .
$$

For $i \in\{1,2,3\}$ the $i$-neighbor of a reduced ideal $\mathbf{a}$ is defined as the ideal $\mathbf{b}=\frac{1}{\alpha} \mathbf{a}$ for $\alpha \in \mathbf{a}$ subject to

$$
|\alpha|_{i}>1, \quad|\alpha|_{j}<1 \quad \text { for } j \in\{1,2,3\} \backslash\{i\}
$$

Received by the editor August 20, 1992.

1991 Mathematics Subject Classification. Primary 11Y40, 11-04, 11R16, 11R27, 11 R29.

Research of the second and third authors was supported by Deutsche Forschungsgemeinschaft. 
and

$$
\left\{\alpha^{\prime} \in \mathbf{a}:\left|\alpha^{\prime}\right|_{i}<\max \left\{1,|\alpha|_{i}\right\}\right\}=\{0\} .
$$

In [1] it is proved that a system of fundamental units can be obtained as follows:

\section{Algorithm.}

Input: maximal order $O_{F}$ of $F$.

Output: system $\left\{\varepsilon_{1}, \varepsilon_{2}\right\}$ of fundamental units.

found $=$ false, $k=1, \mathbf{a}_{1}=o_{F}$.

While not found do

\{

Compute 1-neighbor $\mathbf{a}_{k+1}=\frac{1}{\alpha_{k}} \mathbf{a}_{k}$ of $\mathbf{a}_{k}$.

If there is $l_{1}<k$ with $\mathbf{a}_{l_{1}}=\mathbf{a}_{k+1}$

then found $=$ true,

else $k=k+1$.

\}

found $=$ false, $i=1, \mathbf{b}_{1}=\mathbf{a}_{l_{1}}$.

While not found do

\{

Compute 2-neighbor $\mathbf{b}_{i+1}=\frac{1}{\beta_{i}} \mathbf{b}_{i}$ of $\mathbf{b}_{i}$.

If there is $l_{2} \in\left\{l_{1}, \ldots, k\right\}$ with $\mathbf{a}_{l_{2}}=\mathbf{b}_{i+1}$

then found $=$ true,

else $i=i+1$.

\}

Set

$$
\varepsilon_{1}=\prod_{j=l_{1}}^{k} \alpha_{j} \quad \text { and } \quad \varepsilon_{2}=\prod_{j=1}^{i} \beta_{j} / \prod_{j=l_{1}}^{l_{2}-1} \alpha_{j}
$$

End.

In the following table we show the magnitudes of the regulators

$$
R_{F}:=\left|\operatorname{det}\left(\begin{array}{ll}
\log \left|\varepsilon_{1}^{(1)}\right| & \log \left|\varepsilon_{2}^{(1)}\right| \\
\log \left|\varepsilon_{1}^{(2)}\right| & \log \left|\varepsilon_{2}^{(2)}\right|
\end{array}\right)\right|
$$

in dependence on the Galois group structure:

\begin{tabular}{|r||r|r||r|r|r|r|}
\hline \multicolumn{1}{|c||}{} & \multicolumn{2}{c|}{$\mathrm{D} 4$} & \multicolumn{2}{c|}{ S4 } & \multicolumn{2}{c|}{ \#/frequency } \\
\hline \hline \#/frequency & 9772 & $10.78 \%$ & 80899 & $89.22 \%$ & 90671 & $100 \%$ \\
\hline \hline $0<R_{F}<1$ & 6 & $0.06 \%$ & 9 & $0.01 \%$ & 15 & $0.02 \%$ \\
\hline $1 \leq R_{F}<5$ & 485 & $4.96 \%$ & 347 & $0.43 \%$ & 832 & $0.92 \%$ \\
\hline $5 \leq R_{F}<10$ & 1438 & $14.72 \%$ & 1719 & $2.12 \%$ & 3157 & $3.48 \%$ \\
\hline $10 \leq R_{F}<20$ & 2624 & $26.85 \%$ & 6430 & $7.95 \%$ & 9054 & $9.99 \%$ \\
\hline $20 \leq R_{F}<50$ & 3428 & $35.08 \%$ & 23943 & $29.60 \%$ & 27371 & $30.19 \%$ \\
\hline $50 \leq R_{F}$ & 1791 & $18.33 \%$ & 48451 & $59.89 \%$ & 50242 & $55.41 \%$ \\
\hline
\end{tabular}




\section{Class groups}

Since for the class group computation the same algorithm as in $[3,5]$ was used, we will not present details. The main idea is to compute all prime ideals with norm below the Zimmert bound [9]

$$
M_{F}=\left\lfloor\frac{1}{6.792} \sqrt{-d_{F}}\right\rfloor
$$

and to compute sufficiently many relations between those ideals (see $[6,8])$.

We first show the distribution of noncyclic class groups:

\begin{tabular}{|c|c||r|}
\hline D4 & S4 & $\sum$ \\
\hline \hline 244 & 125 & 369 \\
\hline $0.25 \%$ & $0.15 \%$ & $0.41 \%$ \\
\hline
\end{tabular}

The following table shows the distribution of class numbers:

\begin{tabular}{|r||r|r|r|r||r|r|}
\hline \multicolumn{1}{|c||}{} & \multicolumn{2}{c|}{ D4 } & \multicolumn{2}{c|}{ S4 } & \multicolumn{2}{|c|}{ /frequency } \\
\hline \hline \#/frequency & 9772 & $10.78 \%$ & 80899 & $89.22 \%$ & 90671 & $100 \%$ \\
\hline \hline$h_{F}=1$ & 5199 & $53.20 \%$ & 68533 & $84.71 \%$ & 73732 & $81.32 \%$ \\
\hline$h_{F}=2$ & 2839 & $29.05 \%$ & 9270 & $11.46 \%$ & 12109 & $13.35 \%$ \\
\hline$h_{F}=3$ & 501 & $5.13 \%$ & 1462 & $1.81 \%$ & 1963 & $2.16 \%$ \\
\hline$h_{F}=4$ & 769 & $7.87 \%$ & 1037 & $1.28 \%$ & 1806 & $1.99 \%$ \\
\hline $5 \leq h_{F}<10$ & 420 & $4.30 \%$ & 574 & $0.71 \%$ & 994 & $1.10 \%$ \\
\hline $10 \leq h_{F}<20$ & 42 & $0.43 \%$ & 23 & $0.03 \%$ & 65 & $0.07 \%$ \\
\hline $20 \leq h_{F}$ & 2 & $0.02 \%$ & 0 & $0.00 \%$ & 2 & $0.00 \%$ \\
\hline
\end{tabular}

We finally present the frequency of each class group structure and the corresponding minimal field discriminant (if greater than $-10^{6}$ ):

\begin{tabular}{|r|c||c|c||r|}
\hline$h_{F}$ & $C l_{F}$ & D4 & S4 & $\#$ \\
\hline \hline 1 & 1 & $5199(-275)$ & $68533(-283)$ & 73732 \\
\hline 2 & 2 & $2839(-7975)$ & $9270(-6848)$ & 12109 \\
\hline 3 & 3 & $501(-20975)$ & $1462(-25471)$ & 1963 \\
\hline 4 & 4 & $547(-51207)$ & $916(-54764)$ & 1463 \\
\hline 4 & 2,2 & $222(-83600)$ & $121(-132800)$ & 343 \\
\hline 5 & 5 & $135(-82975)$ & $311(-69128)$ & 446 \\
\hline 6 & 6 & $154(-190400)$ & $110(-137300)$ & 264 \\
\hline 7 & 7 & $48(-165744)$ & $75(-169924)$ & 123 \\
\hline 8 & 8 & $47(-218975)$ & $54(-273491)$ & 101 \\
\hline 8 & 2,4 & $21(-319424)$ & $4(-804875)$ & 25 \\
\hline 9 & 9 & $15(-323975)$ & $20(-326111)$ & 35 \\
\hline 10 & 10 & $14(-451975)$ & $9(-645700)$ & 23 \\
\hline 11 & 11 & $9(-593856)$ & $14(-436227)$ & 23 \\
\hline 12 & 12 & $11(-367975)$ & - & 11 \\
\hline 12 & 2,6 & $1(-995600)$ & - & 1 \\
\hline 13 & 13 & $3(-645056)$ & - & 3 \\
\hline 14 & 14 & $2(-788975)$ & - & 2 \\
\hline 16 & 16 & $2(-328975)$ & - & 2 \\
\hline 20 & 20 & $1(-302975)$ & - & 1 \\
\hline 23 & 23 & $1(-616475)$ & - & 1 \\
\hline \hline$\#$ & & 9772 & 80899 & 90671 \\
\hline
\end{tabular}


The computations were done on Apollo workstations (CPU Motorola 68030), using the number-theoretic program package KANT (see [7]). All data can be obtained from the second author.

\section{BIBLIOGRAPHY}

1. J. Buchmann, A generalization of Voronoi's unit algorithm. I, II, J. Number Theory 20 (1985), 177-209.

2. J. Buchmann, D. Ford, and M. Pohst, Enumeration of quartic fields of small discriminant, Math. Comp. 60 (1993), 873-879.

3. J. Buchmann, M. Pohst, and J. v. Schmettow, On the computation of unit groups and class groups of totally real quartic fields, Math. Comp. 53 (1989), 387-397.

4. H. Cohen and J. Martinet, Class groups of number fields: Numerical heuristics, Math. Comp. 48 (1987), 123-137.

5. M. Pohst and J. Graf v. Schmettow, On the computation of unit groups and class groups of totally complex quartic fields, Math. Comp. 60 (1993), 793-800.

6. M. Pohst and H. Zassenhaus, Algorithmic algebraic number theory, Cambridge Univ. Press, New York, 1989.

7. J. Graf v. Schmettow, KANT - a tool for computations in algebraic number fields, Computational Number Theory (A. Pethö, M. Pohst, H. Williams, and H. G. Zimmer, eds.), de Gruyter, Berlin, 1991, pp. 321-330.

8. ___ Beiträge zur Klassengruppenberechnung, Dissertation, Düsseldorf, 1991.

9. R. Zimmert, Ideale kleiner Norm in Idealklassen und eine Regulatorabschätzung, Invent. Math. 62 (1981), 367-380.

(Buchmann) Fachbereich 14 Informatik, Universität des SaArlandes, Postfach 1150 , D-66041 SAARBRÜCKEN, GERMANY

E-mail address: buchmann@cs.uni-sb.de

(Pohst) Fachbereich 3 Mathematik, Tu Berlin, Strasse des 17. Juni 136, 10623 Berlin, GERMANY

E-mail address: pohst@math.tu-berlin.de

(v. Schmettow) viA PoHST 